\title{
Assessment of Adherence to Guidelines for Hepatocellular Carcinoma Screening in HIV/HCV coinfected patients
}

\section{Jonathan M. Fenkel, MD and Victor J. Navarro, MD}

Division of Gastroenterology and Hepatology, Thomas Jefferson University Hospital, Philadelphia, PA, United States

DISCLOSURES

This investigator-initiated research study was supported by a clinical research grant from Bristol-Myers-Squibb.

\section{BACKGROUND}

Up to $40 \%$ of patients with HIV infection in US are coinfected with Hepatitis C $(\mathrm{HCV})$. $^{1-3}$

Compared to HCV monoinfected patients, coinfected patients have:

Faster progression to cirrhosis 12

hcreased incidence of hepatocellular carcinoma $\left(\mathrm{HCC}^{4}\right.$

Published AASLD guidelines recommend every 6 month ultrasound (US) as the

The majority of gastroenterologists are aware of the AASLD guidelines and apply

Real world surveillance practices among primary providers have not been

\section{OBJECTIVE}

Presuming that a large proportion of care of HIV/HCV coinfected patients is HCC surveillance adherence practices.

\section{METHODS}

25-question survey sent via US Mail.

Study Cohort included all Primary Care and Infectious Diseases physicians in the whose mailong adresses were publicly avalable $(n=3,100)$.

1608 Family Medicine (FM), 1384 General Internal Med (IM)

53 hospitals in 11 counties in four states (PA, NJ, DE, MD) had websites with a
physician locator search function and were included in the study cohort.

The survey measured provider demographics and likelihood of ordering liver imaging in coinfected patients, with and without known cirrhosis.

Adherence was defined as reporting any imaging test (US, CT, or MRI) ordered at 6 month intervals.

\section{RESULTS}

The overall response rate $=12.3 \%(n=387)$

The responding cohort included: 208 FM, 142 IM, 34 ID, 3 Med-Peds

$34(8.7 \%)$ self-identified as HIV specialists (28 ID, 6 IM).

Respondent demographics are outlined in Table 1.

\begin{tabular}{|l|c|}
\hline \multicolumn{2}{|c|}{ Table 1: Respondent Demographics } \\
\hline Median Age (range) & $51.5 \pm 11.6(30-89)$ \\
\hline Median Years in Practice & $20 \pm 12.2(1-61)$ \\
\hline University/Academic & $17.4 \%$ \\
\hline Private Practice & $59.9 \%$ \\
\hline Median Total Patients/Month & $320 \pm 273.8$ \\
\hline Median HIV/HCV Patients/Month & $1 \pm 7.24$ \\
\hline
\end{tabular}

PATIENTS WITH KNOWN CIRRHOSIS

$81.5 \%$ of respondents ( $n=345$ ) reported being somewhat or very likely to order
any liver imaging tests (US, CT, or MRI).

Only $42.3 \%$ were adherent to HCC screening guidelines using any imaging

modality (every 6 months).

No difference in adherence was observed between HIV specialists and non-HIV specialists $(41.2 \%$ vs. $42.4 \%, \mathrm{p}=1.00)$, or with a more liberal yearly screening .

No difference in adherence was observed between University and non-University
physicians $(39.1 \%$ vs. $43.1 \%$, $=0.05)$. physicians (39. $1 \%$ vs. 43.1\%, $p=0.58)$.

Figure 1 delineates the percentage of respondents who reported being somewhat or very likely to order imaging by any modality, categorized by all respondents,
HIV-specialists, and non-HIV specialists. No significant difference was observed between any of the groups

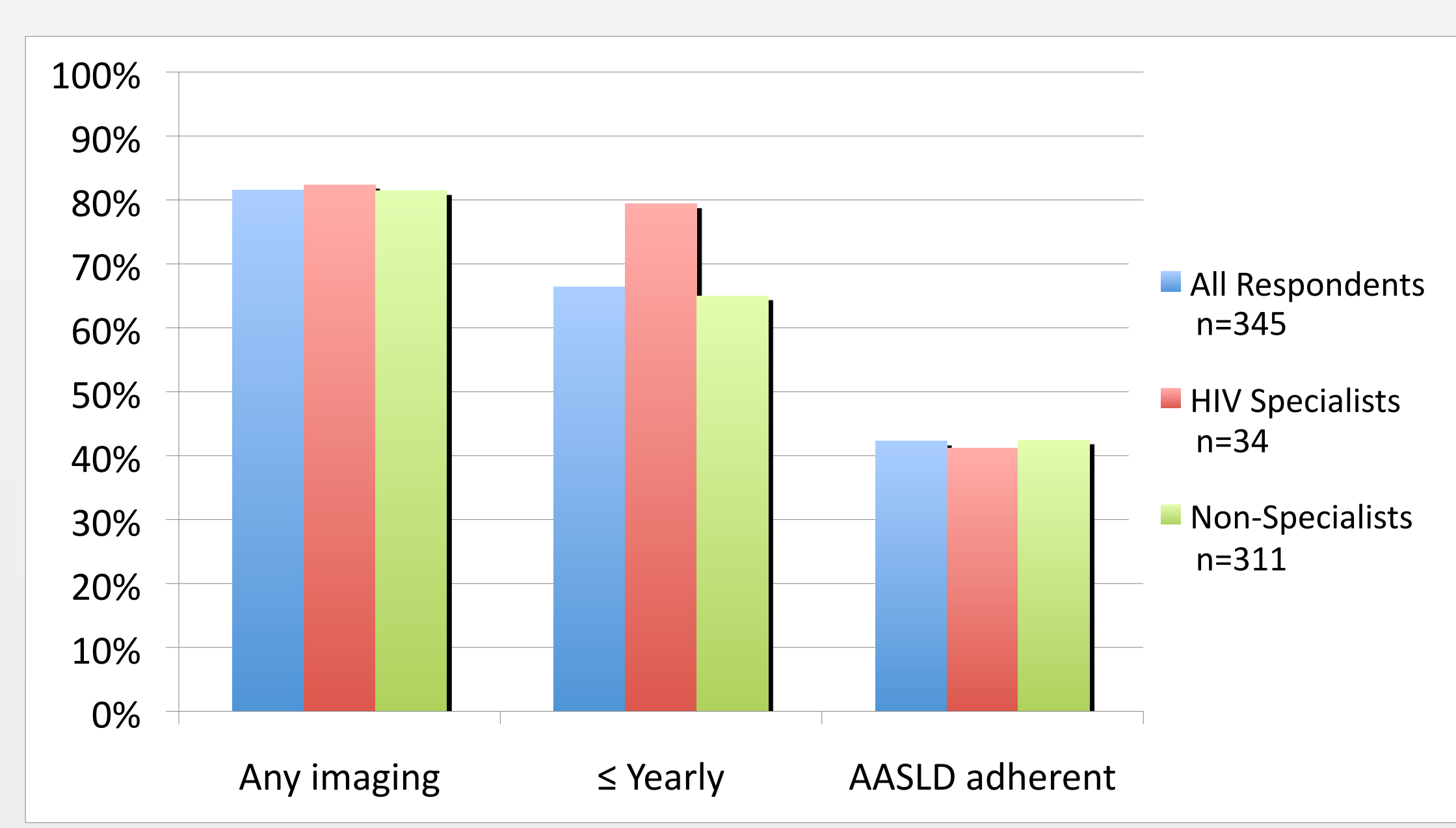

Figure 1: Self-reported frequency of ordering imaging on coinfected cirrhotics
PATIENTS WITHOUT KNOWN CIRRHOSIS

$70.4 \%$ of respondents were somewhat or very likely to order imaging

$$
49.2 \% \text { would likely order at least yearly imaging }
$$

University providers were more likely to order imaging (Figure 2, OR $2.03,95 \% \mathrm{Cl}$
$1.03-398, \mathrm{p}=0.049$ ) but not significantly more likely to order (

HIV specialists were similarly likely to non-HIV specialists to order any imaging

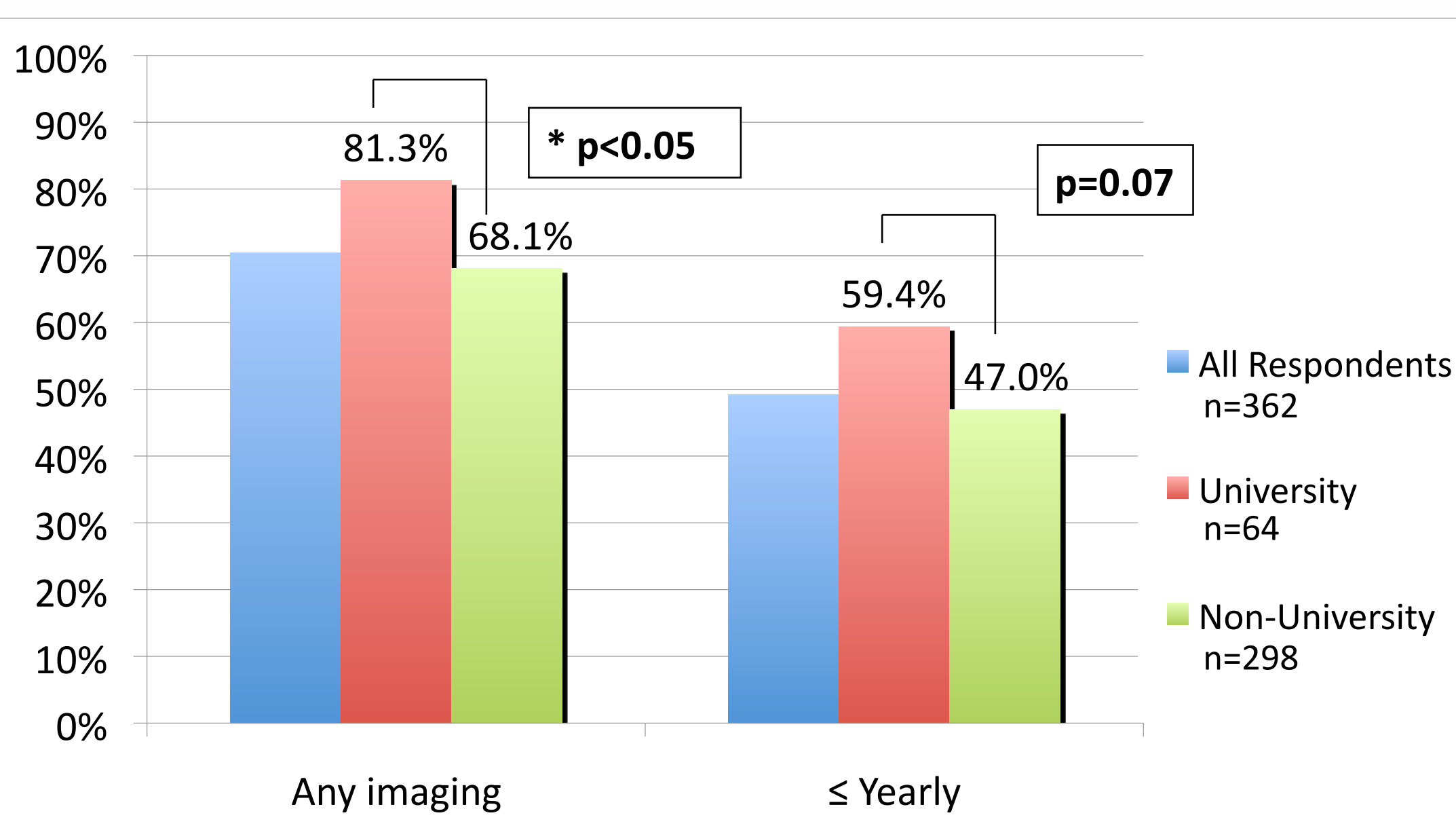

Figure 2: Self-reported frequency of ordering imaging on coinfected patients without known cirrhosis

\section{CONCLUSIONS}

Self-reported adherence with published guidelines for HCC screening is poor among primary providers for HVITCV coinfected patients, including HIV specialists and

Universily-based providers,

Unnecessary imaging is also frequently ordered on non-cirrhotics, particularly by University-based providers.

Improved adherence to guidelines is needed among primary providers as over $50 \%$ of HCC's may be missed, and many patients may not be referred for subspecialty Gl or

\section{REFERENCES}

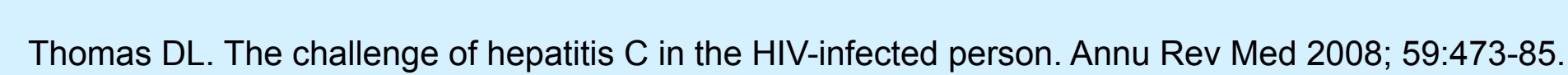

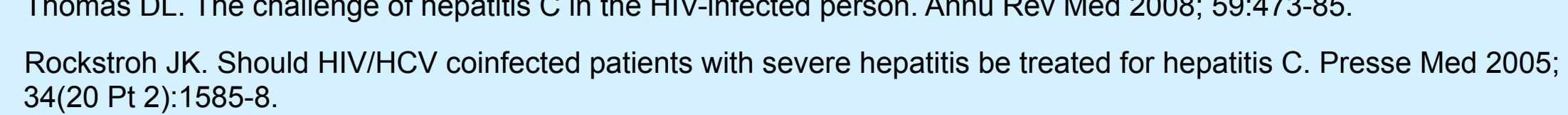

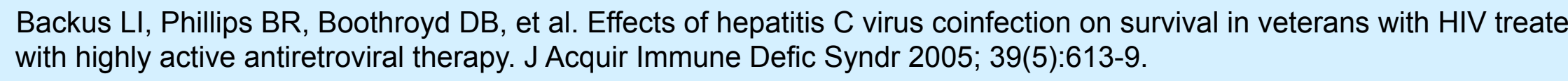

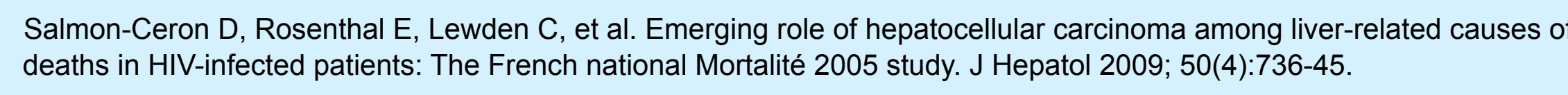

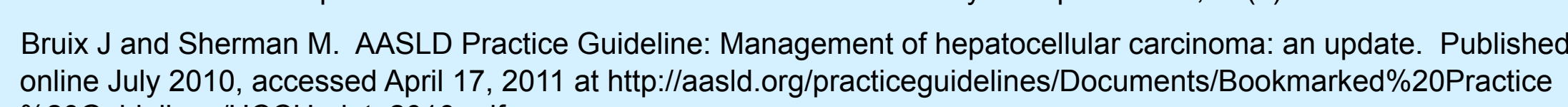

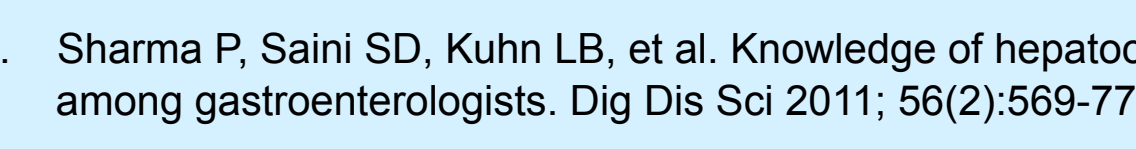

